\title{
Line-by-Line Addressing of RMS Responding Matrix Displays With Wavelets
}

\author{
T. N. Ruckmongathan, V. Arun, and Babu Hemanth Kumar
}

\begin{abstract}
A line-by-line addressing technique that is based on wavelets is proposed to obtain gray shades in root-mean-square responding matrix displays. A large number of gray shades can be displayed with a smaller number of voltages (simple drivers) as compared with amplitude modulation and a smaller number of time intervals as compared with the frame modulation. The technique is demonstrated by displaying 128 gray shades in a liquid crystal display.
\end{abstract}

Index Terms-Gray shades, liquid crystal displays (LCDs), matrix addressing, multiplexing, wavelets.

\section{INTRODUCTION}

A MPLITUDE modulation [1] was the first technique that attempted to display a large number of gray shades. Hardware complexity of the data drivers is high for this technique because $2(G-1)$ voltages are necessary in the data waveforms to display $G$ gray shades. Hardware complexity will be less if analog sample-and-hold type drivers are used instead of the digital drivers, but the power consumption will be high. Pulse height modulation [2] for displaying gray shades is similar to the amplitude modulation and, hence, has the same merits and demerits as the amplitude modulation. The successive approximation techniques [3] can display a large number of gray shades with the same drivers as that of bi-level displays with pixels driven to either ON or OFF states. Hardware complexity of the modified voltage-level generator and a few analog multiplexers that are necessary to achieve gray shades in a bi-level display is not significant as compared with the hardware complexity of the drivers that is necessary to display large number of gray shades with amplitude modulation. Supply voltage of the successive approximation technique increases with the number of gray shades. Wavelets for driving (matrix-addressing) liquid crystal displays (LCDs) was demonstrated [4] recently. Wavelets can be used to reduce the supply voltage and hardware complexity of the drivers [5], [6] in rms responding displays, especially when the number of gray shades is large. All of these addressing techniques are based on selecting several address lines simultaneously while scanning the matrix displays. Our objective is to show that the simple line-by-line addressing can be used to display a large number

Manuscript received December 12, 2006; revised February 22, 2007.

The authors are with the Raman Research Institute, Bangalore 560080, India (e-mail: ruck@rri.res.in; arunv_146@yahoo.co.in; babu-hemanthkumar@yahoo.com).

Color versions of one or more of the figures in this paper are available online at http://ieeexplore.ieee.org.

Digital Object Identifier 10.1109/JDT.2007.900917 of gray shades without much of an increase in the hardware complexity of the drivers and without much of an increase in the supply voltage. A line-by-line addressing that is based on using wavelets is proposed in this paper. Effective utilization of wavelets is illustrated with an example: displaying 128 gray shades in a passive matrix LCD using the Haar wavelets. Select waveform profiles and the orthogonal matrices for displaying 32 and 64 gray shades are also discussed in this paper.

\section{BACKGROUND AND APPROACH TO THE PROBLEM}

LCD is a slow responding device and its electrooptic response does not follow the abrupt changes in the electric field across the pixels. The state of a pixel is determined by the energy delivered to it as long as the period of the addressing waveforms is less as compared with the response times of the display (rms response). Hence, gray shades can be displayed by modulating the energy delivered to the pixels with a set of orthogonal wavelets. For example, the seven Haar wavelets (that are used in our prototype) for displaying 128 gray shades are shown in the following equations:

$$
\begin{aligned}
& \psi_{6}=\{+4,+4,+4,+4,-4,-4,-4,-4\} \\
& \psi_{5}=\{+4,+4,-4,-4,0,0,0,0\} \\
& \psi_{4}=\{0,0,0,0,0,0,+4,-4\} \\
& \psi_{3}=\{0,0,0,0,+2,+2,-2,-2\} \\
& \psi_{2}=\{0,0,0,0,+2,-2,0,0\} \\
& \psi_{1}=\{0,0,+\sqrt{2},-\sqrt{2}, 0,0,0,0\} \\
& \psi_{0}=\{+1,-1,0,0,0,0,0,0\} .
\end{aligned}
$$

These wavelets satisfy the following conditions.

1) Energy of the wavelet is proportional to an integer power of two, so that its energy corresponds to one of the gray shade bit.

2) DC free wavelets are used to ensure long life of the display; life of the LCD is reduced if dc voltages are present across the pixels.

Each one of these wavelets may be used to select an address line and deliver a quantum of energy that is proportional to one bit of the gray shade, and this process could be repeated sequentially to exhaust all of the bits. Then, the number of time intervals is large (56. $N$ to display 128 gray shades) even though it is smaller than (127.N) that of the frame modulation [7] and the pulsewidth modulation [8]. Suppressing the zeros in the wavelets can reduce the number of time intervals to $(24 . N)$. Even then, it is more than that of the wavelet-based addressing 


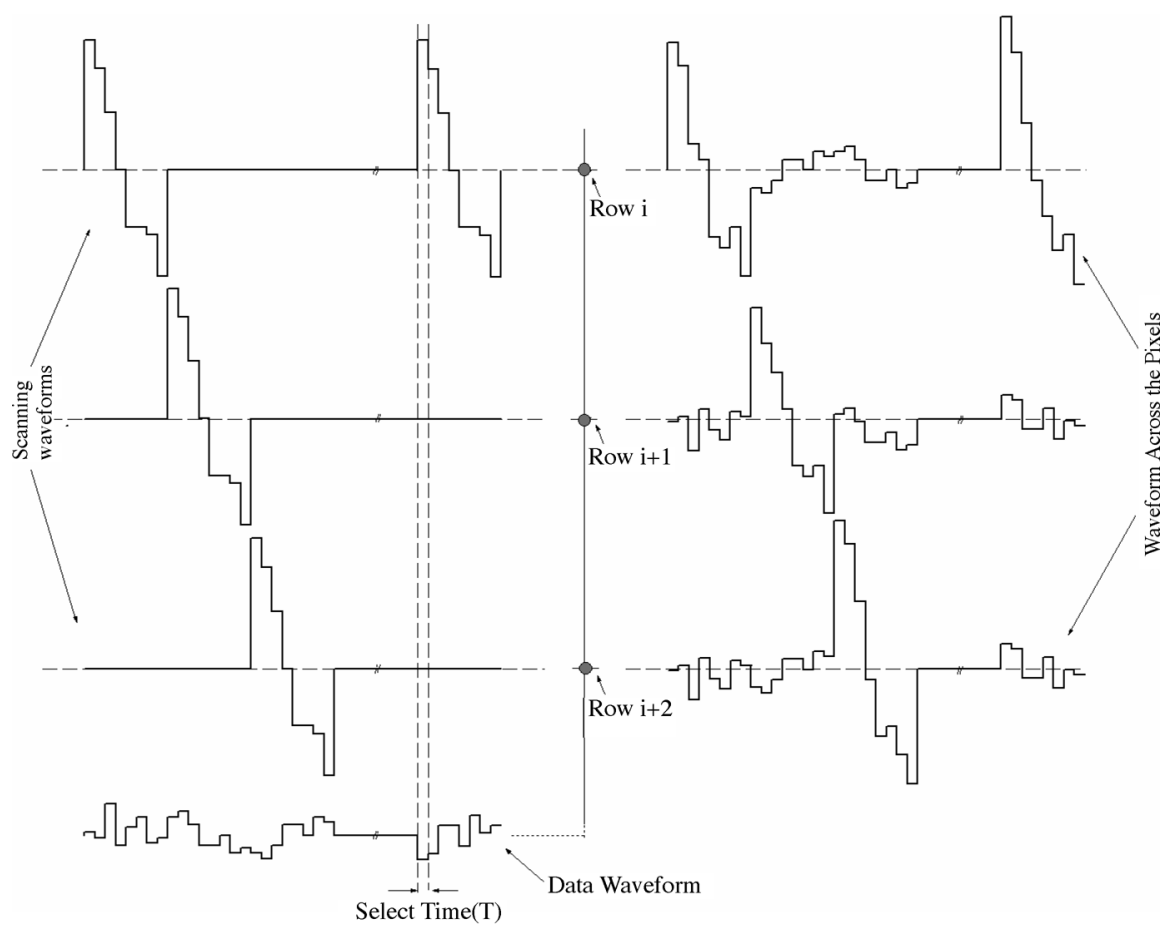

Fig. 1. Typical waveforms when the matrix display is scanned with waveforms derived from wavelets. Scanning (row) waveforms have seven voltages and the column (data) waveforms have 21 voltages.

techniques that are based on selecting several address lines simultaneously [4]-[6]. The number of time intervals to complete a cycle is $(8 . N)$ if these orthogonal wavelets are used for scanning the matrix display by selecting several ( 3 or 4 ) rows simultaneously for displaying 128 gray shades. Our aim is to display a large number of gray shades with line-by-line addressing and yet ensure that the number of time intervals does not exceed that of the multiline addressing. This goal has been achieved by selecting each row with a waveform profile that is obtained by adding all of the wavelets as proposed in Section II. Although the technique is illustrated with the Haar wavelets, other orthogonal wavelets can also be used.

\section{TECHNIQUE}

Consider a matrix display with $N$ address lines and $M$ data lines. Let the gray shade to be displayed in a pixel at the intersection of row "i" and column "j" be

$$
g_{i, j}=\sum_{k=0}^{g-1} d_{i, j, k} .
$$

Here, the index $k$ corresponds to the gray-shade bit (most significant to the least significant) of the "g"-bit data. Let $\Psi_{k}$ be the wavelet that has energy proportional to $2^{k}$, corresponding to the bit- $k$ of the gray-shade data. Let the row "i" of the matrix display be selected with the waveform that is proportional to the waveform that is obtained by adding the "g" wavelets. The expression for the select waveform is given as

$$
\text { Select waveform } \mathrm{i}=V_{r} \cdot \sum_{k=0}^{g-1} \psi_{k}
$$

where $V_{r}$ is the proportionality constant. Hence, the select voltages during the eight time intervals in our example are as follows:

$+9 V_{r},+7 V_{r},+\sqrt{2} V_{r},-\sqrt{2} V_{r}, 0,-4 V_{r},-2 V_{r}$ and $-10 V_{r}$.

The other $(\mathrm{N}-1)$ address lines (nonselected) in the matrix display are held at ground potential. The column waveform that is to be applied to the column " $\mathrm{j}$ " is obtained by summing the products of the value assigned to the data bit and the corresponding wavelets as shown in (11). The value assigned to the bit- $k\left(d_{i, j, k},\right)$ is " +1 " if the bit is "logic- 0 " and a value of " -1 " is assigned to the bit otherwise:

$$
\text { Column waveform }_{\mathrm{j}}=\mathrm{V}_{\mathrm{c}} \cdot \sum_{k=0}^{g-1} \psi_{k} \cdot d_{i, j, k}
$$

where $V_{c}$ is the amplitude of the unit voltage that serves as the constant of proportionality in the column waveforms. Multiplication of the wavelets with +1 or -1 ' reduces to just assigning the appropriate sign to the wavelets $\Psi_{k}$ and summing them. The 


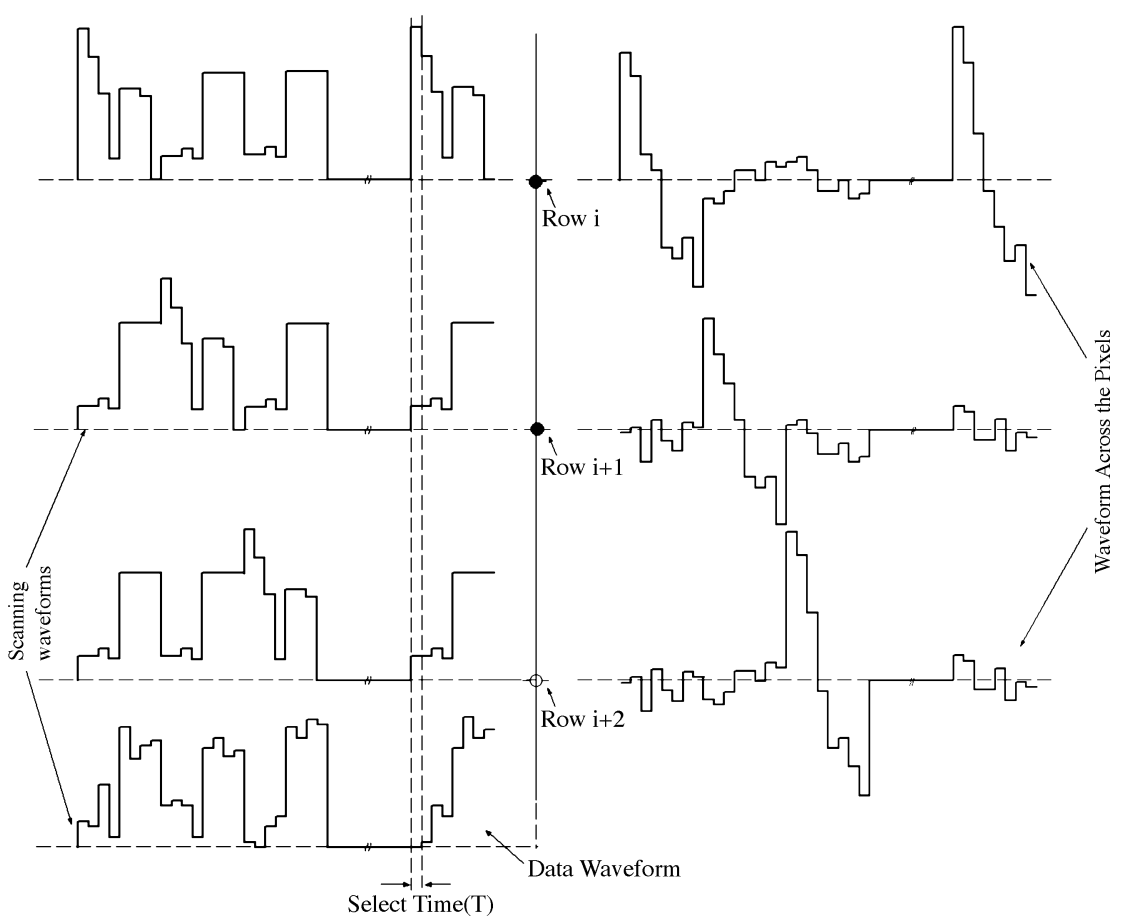

Fig. 2. Typical waveforms when the row (scanning) and column (data) waveforms are modified to reduce the supply voltage. Both the row and column waveforms are shifted by the same amount. Hence, the waveform across the pixels is same as that in Fig. 1 although the row and column waveforms differ from that of Fig. 1.

column waveform can also be obtained by the matrix multiplication by arranging the seven wavelets of (1)-(7) in to a $7 \times 8$ matrix and multiplying its transpose with the data vector, as shown in

$$
\begin{array}{r}
C_{j}(t)=\left[\begin{array}{cccccccc}
+4 & +4 & +4 & +4 & -4 & -4 & -4 & -4 \\
+4 & +4 & -4 & -4 & 0 & 0 & 0 & 0 \\
0 & 0 & 0 & 0 & 0 & 0 & +4 & -4 \\
0 & 0 & 0 & 0 & +2 & +2 & -2 & -2 \\
0 & 0 & 0 & 0 & +2 & -2 & 0 & 0 \\
0 & 0 & +\sqrt{2} & -\sqrt{2} & 0 & 0 & 0 & 0 \\
+1 & -1 & 0 & 0 & 0 & 0 & 0 & 0
\end{array}\right]^{T} \\
\\
\end{array}
$$

At a given instant of time, just one voltage is applied to the selected row, and it is obtained by summing the elements of a column in the matrix of wavelets in (12). The column signal that is proportional to the column voltage is obtained as the dot product of the data with the column of the matrix of orthogonal wavelets. A cycle is complete when all of the address lines in the matrix display are selected once. The display is refreshed fast enough by repeating this cycle to avoid flicker. Waveforms across the pixels will be dc-free as long as all the wavelets are dc-free. In case the orthogonal wavelets are not dc-free, then the dc-free condition can be achieved by reversing the polarity of both the scanning and data waveforms in a periodic manner. Select pulses may be clustered as illustrated in Fig. 1 or it could be distributed in several frames of a cycle. The order of the voltages in the select waveform profile may be changed as long as the one-to-one correspondence among the wavelets and data bits is maintained while computing the column (data) voltage. RMS voltage across the pixels will be same as long as the period of a cycle is less than the response time, but the frequency spectrum across the pixels will vary depending on the distribution of select pulses in a cycle. Hence, it can be used to enhance the performance of the display, like brightness uniformity of pixels or reducing power dissipatio. The select voltages may also be distributed in to several frames to achieve such improvements. Typical waveform profiles for selecting the address lines when the number of gray shades ranges from 32 to 128 are shown in Table I. The corresponding matrices of orthogonal wavelets and the number of voltages in the scanning (row) and the data (column) waveforms are also shown in this table. An analysis of the technique is presented in Section IV.

\section{ANALYSIS}

Energy delivered to a pixel that is located at the intersection of row-i and column-j during a frame when select and data waveforms are applied to the matrix display is given by

$$
\begin{aligned}
& \left(V_{r} \cdot \sum_{k=0}^{g-1} \psi_{k}-V_{c} \cdot \sum_{k=0}^{g-1} \psi_{k} \cdot d_{i, j, k}\right)^{2} \cdot T \\
& \quad+(N-1)\left(V_{c} \cdot \sum_{k=0}^{g-1} \psi_{k} \cdot d_{i, j, k}\right)^{2} \cdot T .
\end{aligned}
$$

The first term in this equation corresponds to the voltage across the pixel when the corresponding address line (row) is selected while the second term corresponds to the data voltages appearing across the pixel when other $(\mathrm{N}-1)$ address lines 
TABLE I

SELECT WAVEForm PROFILES FOR GRAY SHADES

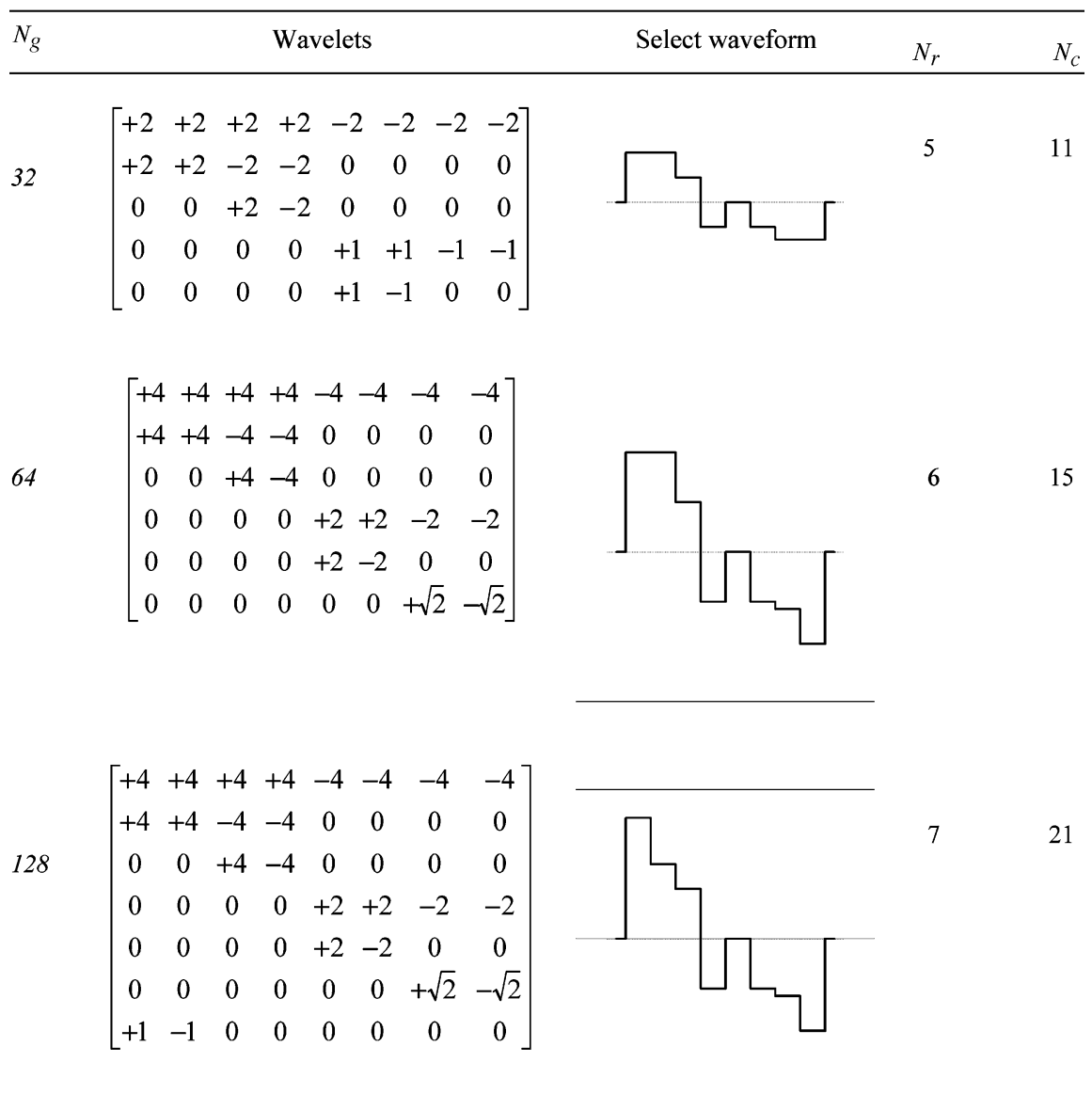

$N_{g}$-number of gray shades; $N_{r}$-number of voltages in row waveforms;

$N_{c}$-number of voltages in the column waveforms

are selected. Orthogonal condition factors like energy of the wavelets and that of data assigned to the pixels viz. (14)-(16) are incorporated into (13) to yield

$$
\begin{aligned}
\int_{0}^{T} \psi_{i} \psi_{j} d t & =0 \quad \forall \quad i \neq j \\
\int_{0}^{T} \psi_{k}^{2} d t & =c .2^{k} \\
d_{i, j, k}^{2} & =+1 \quad \Theta \\
d_{i, j, k} & =\left\{\begin{array}{lll}
+1, & \forall & \text { "logic }-0 " \\
-1, & \forall & \text { "logic }-1 "
\end{array}\right.
\end{aligned}
$$

Hence, the energy delivered to a pixel simplifies to the following expression:

$$
\begin{aligned}
E_{\text {pixel }}= & V_{r}^{2} \cdot \sum_{k=0}^{g-1} \psi_{k}^{2}-2 . V_{r} . V_{c} \ldots \sum_{k=0}^{g-1} \psi_{k}^{2} \cdot d_{i, j, k} \\
& +N \ldots V_{c}^{2} \cdot \sum_{k=0}^{g-1} \psi_{k}^{2}
\end{aligned}
$$

The rms voltage across a pixel is shown as

$$
V_{\mathrm{RMS}}=\sqrt{\frac{c \cdot \sum_{k=0}^{g-1} \psi_{k}^{2}\left(V_{r}^{2}-2 d_{i, j, k} \cdot V_{r} \cdot V_{c}+N V_{c}^{2}\right)}{n_{g} \cdot N}} .
$$

Here, $n_{g}$ is the number of columns in the orthogonal matrix of wavelets. The sign of the mid term $\left(2 . d_{i, j, k} . V_{r} . V_{c}\right)$ in (18) is determined by the "bit- $k$ " of the data. RMS voltage across an ON (all the bits are "logic-1" and they are assigned " -1 ") and OFF (all of the $g$ bits are assigned +1 for "logic-0") pixels is given in the following equations:

$$
\begin{gathered}
V_{\mathrm{ON}}(\mathrm{RMS})=\sqrt{\frac{\sum_{k=0}^{g-1} \psi_{k}^{2}\left(V_{r}^{2}+2 V_{r} V_{c}+N V_{c}^{2}\right)}{n_{g} \cdot N}} \\
V_{\mathrm{OFF}}(\mathrm{RMS})=\sqrt{\frac{\sum_{k=0}^{g-1} \psi_{k}^{2}\left(V_{r}^{2}-2 V_{r} V_{c}+N V_{c}^{2}\right)}{n_{g} \cdot N} .} .
\end{gathered}
$$


The selection ratio, defined as the ratio of rms voltage across ON pixels to that across OFF pixels, is maximum when the following condition is satisfied:

$$
\left.\frac{V_{\mathrm{ON}}}{V_{\mathrm{OFF}}}\right|_{\max .}=\sqrt{\frac{\sqrt{N}+1}{\sqrt{N}-1}}, \quad \text { when } \frac{V_{r}}{V_{c}}=\sqrt{N} .
$$

The expressions for the rms voltage across the ON and OFF pixels get simplified to

$$
\begin{gathered}
V_{\mathrm{ON}}(\mathrm{RMS})=\sqrt{\frac{c \cdot\left(2^{g}-1\right) \cdot 2(N+\sqrt{N}) \cdot V_{c}^{2}}{n_{g} \cdot N}} \\
V_{\mathrm{OFF}}(\mathrm{RMS})=\sqrt{\frac{c \cdot\left(2^{g}-1\right) \cdot 2(N-\sqrt{N}) \cdot V_{c}^{2}}{n_{g} \cdot N}}
\end{gathered}
$$

Contrast in the display will be a maximum when OFF pixels are applied a voltage that is near the threshold voltage of the display. Hence, the unit voltage in the column waveform is determined by equating $\mathrm{V}_{\mathrm{OFF}}$ (RMS) to the $\mathrm{V}_{\mathrm{th}}$ threshold voltage of the electrooptic characteristics of an LCD as follows:

$$
V_{c}=\sqrt{\frac{n_{g} \cdot N}{2 \cdot c \cdot\left(2^{g}-1\right) \cdot(N-\sqrt{N})}} \cdot V_{\text {threshold }} \cdot
$$

Supply voltage of the drive electronics is determined by the maximum swing in the addressing waveforms. Let $P . V_{r}$ be the peak-to-peak amplitude of the row waveforms, and then the supply voltage of the addressing technique (normalized to the threshold voltage of the electrooptic characteristics of the LCD) is

$$
\begin{aligned}
V_{s}\left(W_{-} A P T\right)= & P \cdot V_{r}=P \cdot \sqrt{N} \cdot V_{c} \\
V_{s}\left(W_{-} A P T\right)= & \frac{P}{2} \cdot \sqrt{\frac{n_{g}}{c \cdot\left(2^{g}-1\right)}} \\
& \cdot\left(\frac{2 \sqrt{N}}{\sqrt{2 \cdot(1-(1 / \sqrt{N}))}}\right)
\end{aligned}
$$

Supply voltage of the successive approximation technique [3] that is also based on the line-by-line addressing (SA-APT) is given as follows:

$$
V_{s}\left(S A_{-} A P T\right)=\sqrt{\frac{g \cdot 2^{(g-1)}}{2^{g}-1}} \cdot\left(\frac{2 \sqrt{N}}{\sqrt{2 \cdot(1-(1 / \sqrt{N}))}}\right) .
$$

Supply voltages of these two techniques are compared using the following ratio:

$$
\frac{V_{s}\left(W_{-} A P T\right)}{V_{s}\left(S A_{-} A P T\right)}=\frac{P}{2} \cdot \sqrt{\frac{n_{g}}{c . g \cdot 2^{(g-1)}}} .
$$

\section{REducing THE Supply Voltage}

A technique, similar to that proposed by Kawakami et al. [9] to reduce the supply voltage of the conventional line-by-line ad- dressing can be adopted to reduce the supply voltage of the drive electronics. Let us consider the example of the waveforms for displaying 128 gray shades. The voltages in the select waveform starting from the first to the eighth time intervals in order (a profile that is slightly different from that of (10) is chosen here) are

$$
\begin{aligned}
& +9 V_{r},+7 V_{r},+4 V_{r},-4 V_{r}, 0,-4 V_{r}, \\
& -(6-\sqrt{2}) V_{r} \text { and }-(6+\sqrt{2}) V_{r} .
\end{aligned}
$$

Data voltages in the first and second time intervals are

$$
\pm 9 V_{c}, \pm 7 V_{c} \text { and } \pm V_{c} \text {. }
$$

Data voltages in the third and fourth time intervals are

$$
\pm 12 V_{c} \text { and } \pm 4 V_{c} \text {. }
$$

Similarly, the data voltages in the fifth and sixth time intervals are

$$
\pm 8 V_{c}, \pm 4 V_{c} \text { and } 0
$$

Finally, the data voltages in the seventh and eighth time intervals are

$\pm(6+\sqrt{2}) V_{c}, \pm(6-\sqrt{2}) V_{c}, \pm(2+\sqrt{2}) V_{c}$ and $\pm(2-\sqrt{2}) V_{c}$.

The maximum amplitude of instantaneous voltages across any pixel is $9\left(V_{r}+V_{c}\right)$. Hence, the supply voltage can be reduced if both row and column waveforms are shifted by:

1) $\left(9 V_{c}\right)$ during the first two intervals;

2) $\left(4 V_{r}+12 V_{c}\right)$ for the third to sixth time intervals;

3) $8 V_{r}$ during the last two time intervals.

Then, the voltages in the select waveform are modified as follows:

$$
\begin{aligned}
& +9\left(V_{r}+V_{c}\right),+\left(7 V_{r}+9 V_{c}\right),+\left(8 V_{r}+12 V_{c}\right), \\
& +\left(12 V_{c}\right),+\left(4 V_{r}+12 V_{c}\right),+\left(12 V_{c}\right),+(2+\sqrt{2}) V_{r} \\
& \quad \text { and }+(2-\sqrt{2}) V_{r}
\end{aligned}
$$

The modified data voltages are as follows.

In the first and second time intervals:

$$
+18 V_{c}, 0,+16 V_{c}, 2 V_{c},+10 V_{c} \text { and }+8 V_{c} .
$$

In the third and fourth time intervals, we have

$$
\begin{aligned}
& +\left(4 V_{r}+24 V_{c}\right),+\left(4 V_{r}\right), \\
& +\left(4 V_{r}+16 V_{c}\right) \text { and }+\left(4 V_{r}+8 V_{c}\right) .
\end{aligned}
$$

In the fifth and sixth intervals, we have

$$
\begin{aligned}
& +\left(4 V_{r}+20 V_{c}\right),+\left(4 V_{r}+4 V_{c}\right)+\left(4 V_{r}+16 V_{c}\right), \\
& +\left(4 V_{r}+8 V_{c}\right) \text { and }+\left(4 V_{r}+12 V_{c}\right) .
\end{aligned}
$$

The data voltages in the last two intervals are

$$
\begin{aligned}
& \left(8 V_{r} \pm(6+\sqrt{2}) V_{c}\right),\left(8 V_{r} \pm(6-\sqrt{2}) V_{c}\right), \\
& \left(8 V_{r} \pm(2+\sqrt{2}) V_{c}\right) \text { and }\left(8 V_{r} \pm(2-\sqrt{2}) V_{c}\right) .
\end{aligned}
$$


TABLE II

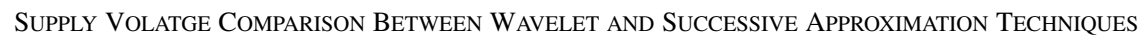

\begin{tabular}{|c|c|c|c|c|}
\hline$N_{g}$ & $\begin{array}{l}\text { Peak to peak voltage in } \\
\text { the scanning waveforms } \\
\left(\mathrm{V}_{\mathrm{p}_{-} \mathrm{APT}}\right)\end{array}$ & $\begin{array}{c}\text { Reduced peak to peak } \\
\text { voltage in the addressing } \\
\text { waveforms }\left(\mathrm{V}_{\mathrm{p}_{-} \text {IAPT }}\right)\end{array}$ & $\frac{V_{s}\left(W_{-} A P T\right)}{V_{s}\left(S A_{-} A P T\right)} \times 100 \%$ & $\frac{V_{s}\left(W_{-} I A P T\right)}{V_{s}\left(S A_{-} I A P T\right)} \times 100 \%$ \\
\hline 32 & $7 V_{r}$ & $4\left(V_{r}+V_{c}\right)$ & 78.26 & 89.44 \\
\hline 64 & $(14+\sqrt{2}) V_{r}$ & $8\left(V_{r}+V_{c}\right)$ & 78.66 & 81.65 \\
\hline 128 & $(15+\sqrt{2}) V_{r}$ & $9\left(V_{r}+V_{c}\right)$ & 77.55 & 85.04 \\
\hline
\end{tabular}

The voltage transformation is not unique, the transformation described in the previous paragraphs is well suited for LCD drivers with the lowest voltage as the common rail, and all other voltages in the addressing waveforms are positive with reference to this common voltage. An example of the voltage transformation that is better suited for the LCD drivers with most positive voltage as the common rail is outlined next. Both the row and column waveforms are shifted as follows.

1) Down by $9 V_{r}$ during the first two time intervals.

2) Down by $4 V_{r}$ during the third to sixth time intervals.

3) Down by $1.5 V_{r}$ during the last two time intervals.

Then, the voltages in the select waveform get modified as follows:

$$
\begin{aligned}
& 0,-2 V_{r}, 0,-8 V_{r},-4 V_{r},-8 V_{r}, \\
& -(7.5-\sqrt{2}) V_{r} \text { and }-(7.5+\sqrt{2}) V_{r}
\end{aligned}
$$

The modified data voltages are as follows.

In the first and second time intervals, we have

$$
-\left(9 V_{r} \pm 9 V_{c}\right),-\left(9 V_{r} \pm 7 V_{c}\right) \text { and }-\left(9 V_{r} \pm V_{c}\right) .
$$

In the third and fifth time intervals, we have

$$
-\left(4 V_{r} \pm 12 V_{c}\right) \text { and }-\left(4 V_{r} \pm 4 V_{c}\right) .
$$

In the fourth and sixth interval, we have

$$
-\left(4 V_{r} \pm 8 V_{c}\right),-\left(4 V_{r} \pm 4 V_{c}\right) \text { and }-4 V_{r}
$$

The data voltages in the last two intervals are

$$
\begin{aligned}
& -\left(1.5 V_{r} \pm(6+\sqrt{2}) V_{c}\right),-\left(1.5 V_{r} \pm(6-\sqrt{2}) V_{c}\right), \\
& -\left(1.5 V_{r} \pm(2+\sqrt{2}) V_{c}\right) \text { and }-\left(1.5 V_{r} \pm(2-\sqrt{2}) V_{c}\right) .
\end{aligned}
$$

The number of voltages in the scanning waveform increases from 7 to 9 (or 10) as compared with 3 (or 4 ) in the conventional line-by-line addressing. The nonselect voltages when the common rail is the most negative voltage are $+9 V_{c}$, $\left(4 V_{r}+12 V_{\mathrm{c}}\right)$, and $8 V_{r}$. Similarly, the nonselect voltages when the most positive voltage is the common rail are $-9 V_{r},-4 V_{r}$ and $-1.5 V_{r}$. It is important to note that the number of voltages in the data waveforms does not change where as it doubles (from two to four voltages) in the conventional addressing technique. Supply voltage will be proportional to $9\left(V_{r}+V_{c}\right)$

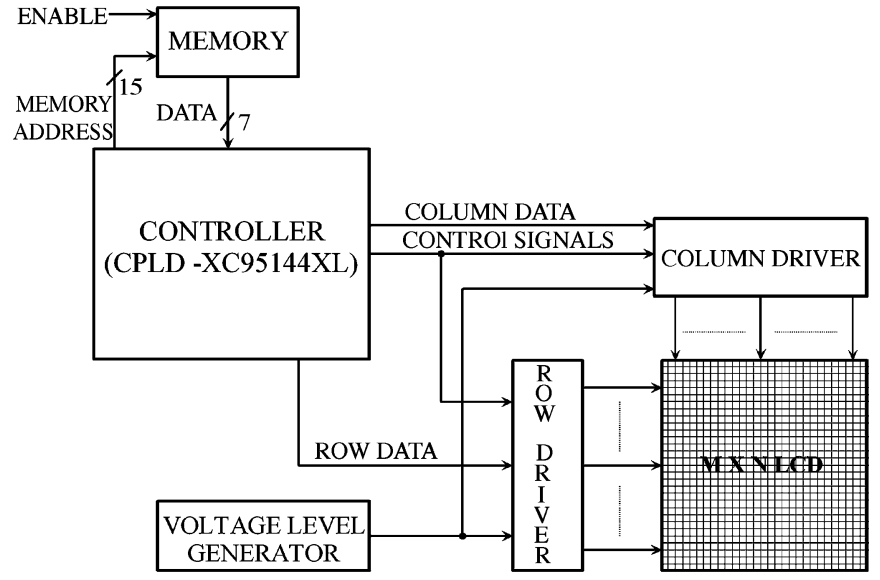

Fig. 3. Block diagram of the prototype of a $32 \times 32$ matrix LCD that is scanned using a line-by-line addressing technique that is based on wavelets.

as compared with $(15+\sqrt{2}) V_{r}$. The percentage reduction in supply voltage due to modification in the addressing waveforms is

$$
\frac{V s\left(W \_I A P T\right)}{V s\left(W_{-} A P T\right)}=\left(1-\frac{9(\sqrt{N}+1)}{(15+\sqrt{2}) \sqrt{N}}\right) \cdot 100 \approx 45 \% .
$$

Typical waveforms when the row and column waveforms that are shifted to reduce the supply voltage of the driver circuit is shown in Fig. 2.

The modification of the addressing waveforms is not unique; the shift in the addressing waveforms has to be such that the difference among these voltages is equal to the maximum voltage across the pixels. For example, it is sufficient to ensure that all of the voltages in the scanning and data waveforms lie with in the voltage range of $9\left(V_{r}+V_{c}\right)$, which is the maximum voltage across the pixel during a cycle. Supply voltages of the wavelet-based line-by-line addressing with modified waveforms $\left(\mathrm{V}_{\mathrm{s}}\left(\mathrm{W} \_\mathrm{IAPT}\right)\right)$ and the successive approximation technique based on the line-line-addressing with modified waveforms $\left(\mathrm{V}_{\mathrm{s}}(\mathrm{SA} I A P T)\right)$ are also compared in Table II.

\section{DRIVE ELECTRONICS}

A block diagram of the prototype is shown Fig. 3. Voltages in the addressing waveforms are generated using a resistor network. The number of voltages in the addressing waveform decides the hardware complexity of the drivers. The number of voltages in the scanning waveform ranges from 6 to 11 as the number of gray shades is increased from 8 to 128 . However, at a given instant of time, just two voltages viz., select and 
TABLE III

COMPARISON OF THE GRAY-SHADE TECHNIQUeS (128 GRAY SHADES)

\begin{tabular}{cccc}
\hline Parameter & $\begin{array}{c}\text { Amplitude modulation and } \\
\text { PHM (line-by-line) technique }\end{array}$ & $\begin{array}{c}\text { Successive approximation } \\
\text { technique }\end{array}$ & Wavelet based technique \\
\hline $\begin{array}{c}\text { Number of time intervals for } \\
128 \text { gray shades }\end{array}$ & $4 \mathrm{~N}$ & $14 \mathrm{~N}$ & $8 \mathrm{~N}$ \\
Supply voltage & Low & High & Intermediate \\
$\begin{array}{c}\text { Number of voltages in } \\
\text { scanning waveforms }\end{array}$ & 3 & $\begin{array}{c}15 \\
(22 \text { for IAPT })\end{array}$ & (10 for IAPT) \\
$\begin{array}{c}\text { Number of voltages in the } \\
\text { data waveforms }\end{array}$ & 254 & 14 & 21 \\
\end{tabular}

* IAPT: Improved Alt and Pleshko Technique

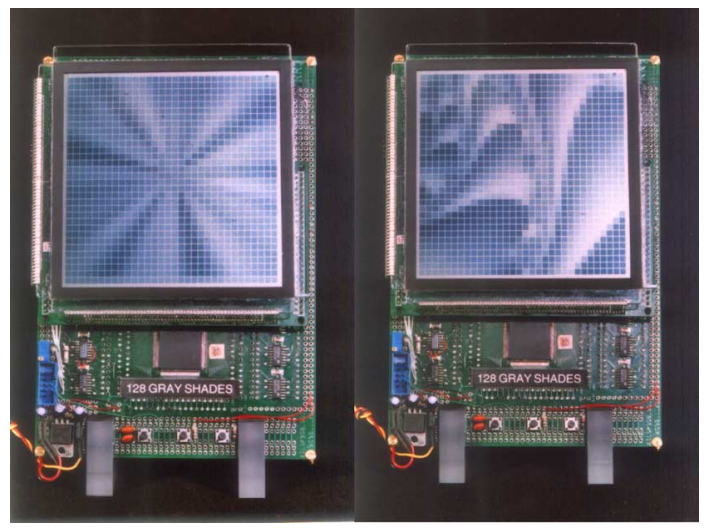

Fig. 4. Photographs of a $32 \times 32$ matrix LCD driven by the wavelet-based line-by-line addressing technique. It is capable of displaying 128 gray shades.

nonselect voltages are necessary for scanning the matrix display. Hence, the hardware complexity is the same as that of the conventional line-by-line addressing for displaying bi-level images. LCD drivers like the HD-44100 can be used along with a few multiplexers to select and route the appropriate voltages from the voltage-level generator. The number of voltages in the data waveforms is at least 21 for displaying 128 gray shades, as shown in Table I. It demands a display driver having 5-bit shift register, 5-bit latch, and an analog multiplexer (21:1) for each output. The number of voltages is limited to just eight at any instant of time, and a good reduction in hardware complexity of the driver can be achieved when this observation is incorporated in the design. LCD drivers with 3-bit shift register, 3-bit latch, and analog multiplexers (8:1) for each output are adequate as data drivers (for example, TMS57206). Hence, a 40\% reduction in shift register and latches and 58\% reduction in the analog switches in the multiplexers are achieved. The number of stages in the column driver is equal to the number of columns and hence this reduction is substantial. The reduction is about $62 \%$ for the shift registers and latches $96 \%$ for the analog switches of the output stage of the drivers if the technique is compared with amplitude modulation for the same number of gray shades. Although the column voltages are computed as the dot product

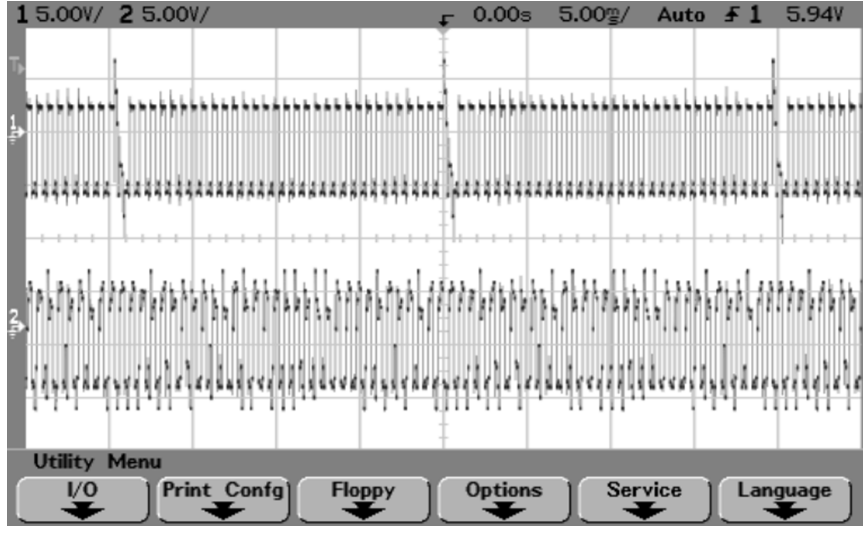

Fig. 5. Typical scanning (upper trace) and data (lower trace) waveforms of the wavelets-based line-by-line addressing technique.

of the select pattern and the corresponding data vectors, it is not necessary to compute them on the fly; they can be computed once and they can be incorporated in to the voltage level generator. Analog multiplexers in the driver circuit are used to apply these voltages depending on the select voltage and the data. The controller is designed to select one row at a time with all the eight row select voltages. During each select voltage, the voltages corresponding to the data of the pixels are applied on the column side. The pixel values (data) are stored in an EPROM in continuous memory locations as a one-dimensional array. The addresses corresponding to the data of the pixels in the selected row are generated repeatedly for all the select voltages (once per each select voltage). Similarly, all the other rows are selected one at a time and the display is refreshed continuously. The controller was implemented using a CPLD (complex programmable logic device). A photograph of the prototype is shown in Fig. 4. Typical row and column (data) waveforms are shown in Fig. 5. A typical waveform across a pixel is shown in Fig. 6.

\section{COMPARISON}

Amplitude modulation [1] and the very similar pulse-height modulation [2] can display 128 gray shades in $(4 . N)$ time intervals with 254 voltages in the column (data) waveforms and three voltages in the scanning waveforms. It may be more appropriate 


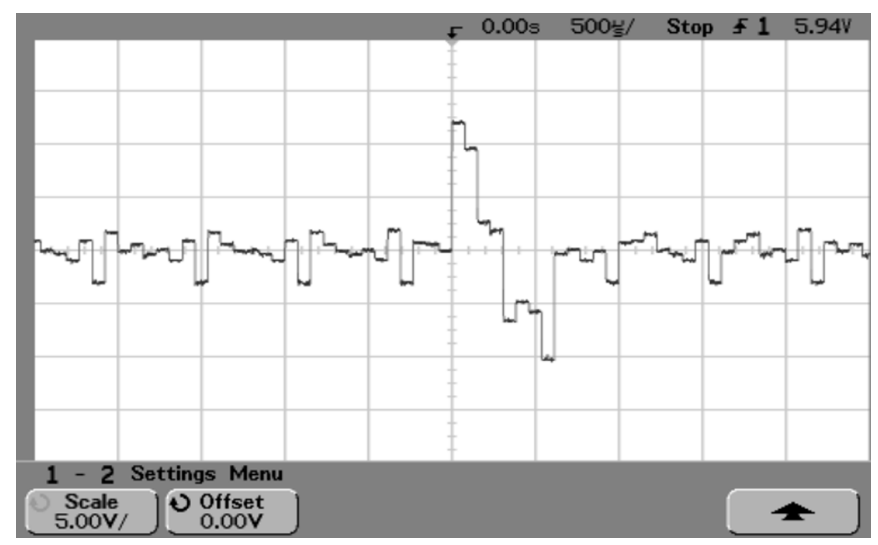

Fig. 6. Waveform across a pixel in the display (part of a cycle is shown here for the sake of clarity).

to compare the wavelet-based technique with the successive approximation technique [3] since both techniques are based on delivering energies that are proportional to the bit-weight of the gray-shade data in several time intervals. A comparison of the wavelet-based technique with amplitude modulation and successive approximation based on line-by-line addressing is given in Table III.

\section{CONCLUSION}

The number of time intervals to complete a cycle is less in the wavelet-based technique as compared to the successive approximation technique when all other parameters are equal. Slow scanning is helpful to reduce the power consumption [10]. The brightness nonuniformity of pixels due to distortion in the addressing waveforms will also be less because the select time is larger when the number of time intervals is small. A lower supply voltage of the wavelet-based technique is advantageous in portable devices. It has been achieved with out increasing the hardware complexity of the row drivers and a moderate increase in hardware complexity of the column drivers. Increasing the number of voltages in the column waveform by a factor of four has paved way to an increase in number of gray shades by factor 64; when wavelet-based addressing is used for scanning the matrix LCD.

\section{ACKNOWLEDGMENT}

The authors would like to thank A. R. Shashidhara for fabricating the LCD and his support in hardware implementation of the prototype.

\section{REFERENCES}

[1] T. N. Ruckmongathan, "Addressing techniques for RMS responding LCDs-A review," in Proc. 12th Int. Display Res. Conf. (Japan Display'92), 1992, pp. 77-80.

[2] A. R. Conner and T. J. Scheffer, "Pulse height modulation (PHM) gray shade method for passive matrix LCDs," in Proc. 12th Int. Display Res. Conf. (Japan Display'92), 1992, pp. 69-72.

[3] T. N. Ruckmongathan, "A successive approximation technique for displaying gray shades in liquid crystal displays (LCDs)," IEEE Trans. Image Process., vol. 16, no. 2, pp. 554-561, Feb. 2007.

[4] T. N. Ruckmongathan, P. Nanditha Rao, and A. Prasad, "Wavelets for displaying gray shades in LCDs," in SID 05 Dig., 2005, pp. 168-171.

[5] T. N. Ruckmongathan, U. Manasa, R. Nethravathi, and A. R. Shashidhara, "Integer wavelets for displaying gray shades in RMS responding displays," J. Display Technol., vol. 2, no. 3, pp. 292-299, Sep. 2006.

[6] T. N. Ruckmongathan, D. Nadig, and P. R. Ranjitha, "Gray shades in RMS responding displays with wavelet based on the slant transforms," IEEE Trans. Electron Devices, vol. 54, no. 4, pp. 663-670, Apr. 2007.

[7] Y. Suzuki, M. Sekiya, K. Arai, and A. Ohkoshi, "A liquid crystal image display," in SID-'83 Digest, Proc. Int. Symp. Soc. Inf. Display, 1983, pp. 32-33.

[8] H. Kawakami, H. Hanmura, and E. Kaneko, "Brightness uniformity in liquid crystal displays," in Proc. Int. Symp. Soc. Inf. Display, 1980, pp. $28-29$.

[9] H. Kawakami, Y. Nagae, and E. Kaneko, "Matrix addressing technology of twisted nematic displays," in SID-IEEE Rec. Biennial Display Conf., 1976, pp. 50-52.

[10] T. N. Ruckmongathan, M. Govind, and G. Deepak, "Reducing power consumption in liquid crystal displays," IEEE Trans. Electron Devices, vol. 53, no. 7, pp. 1559-1566, Jul. 2006.

T. N. Ruckmongathan received the B.E. degree in electronics and communication from the University of Madras, Chennai, India, in 1976, and the M.E and $\mathrm{Ph} . \mathrm{D}$. degrees in electrical communication engineering from the Indian Institute of Science, Bangalore, India, in 1978 and 1988, respectively.

He is currently a Professor with the Raman Research Institute, Bangalore. He was a Visiting Professor with the Chalmers University of Technology, Swedenm during 1998, a Guest Researcher with the Asahi Glass Company R\&D, Yokohama, Japan, during 1991-1993, and an LCD Specialist with Philips, Heerlen, The Netherlands, during 1989-1991. His pioneering work on multi-line addressing techniques, "A generalized addressing technique for RMS responding LCDs," was presented at the International Display Research Conference in 1988 at San Diego. The analysis presented in this paper holds good for most of the multiline addressing techniques. His research work on multiline addressing has been cited in 91 U.S. patents. His main interest is in research and development of new addressing techniques for driving matrix LCD.

Prof. Ruckmongathan is a member of the Society for Information Display.

V. Arun received the B.E. degree in electronics and communication from the Visvesvaraya Technological University, Belgaum, India, in 2006. He is currently a visiting student at the Raman Research Institute, Bangalore, India.

Mr. Arun is a student member of the Society for the Information Display.

Babu Hemanth Kumar received the B.E. degree in electronics and communication from the Visvesvaraya Technological University, Belgaum, India, in 2006. He is currently a visiting student at the Raman Research Institute, Bangalore, India. 